\title{
Comparação e aplicação de dois modelos matemáticos no estudo do metabolismo de fósforo em suínos ${ }^{1}$
}

\author{
José Aparecido Moreira ${ }^{2}$, Dorinha Miriam Silber Schmidt Vitti ${ }^{3}$, Alexandre de Oliveira \\ Teixeira ${ }^{4}$, João Batista Lopes $^{5}$
}

\footnotetext{
1 Projeto financiado pela FAPESP-06/57574-5 e 04/14532-5.

2 Escola Agrícola de Jundiaí-UFRN-Natal-RN.

${ }^{3}$ Laboratório de Nutrição Animal-CENA/USP-Piracicaba-SP

${ }^{4}$ Departamento Técnico-BUNGE Fertilizantes S/A.

${ }^{5}$ Departamento de Zootecnia-UFPI-Teresina-PI.
}

RESUMO - Avaliaram-se dois modelos matemáticos no estudo do fluxo de fósforo no metabolismo de suínos com os objetivos de destacar o modelo mais eficiente e indicar a fonte de fósforo com maior potencial para uso na nutrição de suínos. Foram usados 21 suínos, híbridos machos castrados com peso médio inicial de $32 \mathrm{~kg}$, alimentados com dietas formuladas à base de milho, farelo de soja e óleo vegetal, suplementadas com fosfato bicálcico; fosfato monobicálcico; superfosfato triplo; superfosfato simples; ácido fosfórico ou fosfato de rocha catalão como fontes de fósforo. O delineamento experimental foi o de blocos casualizados, com sete dietas e três repetições, com um animal por unidade experimental. Para o desenvolvimento dos dois modelos, foram utilizados dados de metabolismo e cinética de fósforo nos tecidos, obtidos pela técnica de diluição isotópica. Os modelos apresentaram similaridade e eficiência na determinação das estimativas do fluxo de fósforo nos compartimentos anatômicos. O superfosfato simples e o fosfato bicálcico têm maior potencial para uso em dietas para suínos em crescimento. Entretanto, o fosfato bicálcico apresenta melhor ajuste nos modelos e serve como padrão para nutrição de suínos em crescimento.

Palavras-chave: diluição isotópica, fósforo endógeno, metabolismo, modelo matemático, radiofósforo

\section{Comparison and application of two mathematical models in the study of the phosphorus in pig metabolism}

\begin{abstract}
The aim in this work was to evaluate two mathematical models on the study of the flow of the phosphorus in metabolism of swines aiming at highlighting the most efficient model and indicating the highest potential phosphosrus source to be used in nutrition for swines. It was used 21 hybrid barrow at $32 \mathrm{~kg}$ initial weight fed diets based on corn, soybean meal and vegetal oil, supplemented with dicalcium phosphate, monodicalcium phosphate, triple superphosphate, simple superphosphate, Catalão rock phosphate or phosphoric acid as sources of phosphorus. The experiment was arranged in randomized blocks, with seven treatments and three replicates and one animal per experimental unity. Metabolism data and kinetics of phosphorus in tissues obtained by the isotopic dilution technique were used for development of the two models. The models presented similarity and efficiency in the determination of the estimates of the phosphorus flow in the anatomical compartments. Simple superphosphate and dicalcium phosphate have more potential to be used in growing swines. However, dicalcium phosphate presentes better adjustment in the models and it can be used as a standard for nutrition of growing swines.
\end{abstract}

Key Words: endogenous phosphorus, isotopic dilution, mathematical model, metabolism, radiophosphorus

\section{Introdução}

A suplementação adequada de fósforo em dietas para suínos, além de evitar gastos desnecessários decorrentes de desperdícios, pode contribuir também para aumentar o desempenho do rebanho, assim como o fortalecimento das estruturas ósseas. Além disso, pode proporcionar economia tanto para os produtores como para os fabricantes de ração e pode reduzir no meio ambiente, a descarga de poluentes, que acompanha os dejetos dos animais.

Muitos estudos já foram desenvolvidos com fósforo para avaliar as fontes alternativas, as exigências nutricionais e a adição de enzima fitase (Teixeira et al., 2004a, Sheng et al., 2006; Moreira et al., 2009ab). O surgimento de modelos matemáticos para o estudo do metabolismo animal tem permitido a exploração mais eficiente dos dados obtidos 
em experimentos com o uso da técnica de diluição isotópica, pela qual se usa o fósforo radioativo ${ }^{32} \mathrm{P}$ como traçador.

O modelo do fluxo biológico de fósforo caracteriza-se por uma equação ou um conjunto de equações que simulam o comportamento de um sistema biológico. A utilização de modelos, principalmente com radioisótopo, permite estudar fenômenos complexos, com precisão, por possibilitar o acompanhamento detalhado das reações e dos mecanismos biológicos ou naturais envolvidos.

Experimentos têm sido desenvolvidos no Brasil com suínos na linha de metabolismo de fósforo pela técnica de diluição isotópica desde 1998 e isso tem possibilitado evolução gradativa na qualidade das observações, de modo que atualmente são realizados estudos detalhados do metabolismo de fósforo com uso de modelos matemáticos.

Vitti et al. (2000) desenvolveram um modelo matemático para estudar o fluxo de fósforo no metabolismo de ovinos, que posteriormente foi utilizado no estudo do metabolismo desse mineral em suínos (Teixeira et al., 2004b) e, embora tenha sido desenvolvido em estudo com ovinos, pareceu ser eficiente no estudo com suínos.

Lopes et al. (2001) estudaram o modelo proposto por Fernandes (1995) e propuseram modificações na sua estrutura, introduzindo o compartimento denominado tecidos moles. Com as adaptações desenvolvidas neste modelo, pode-se efetuar estudos detalhados do fluxo e refluxo de fósforo no trato digestivo, na corrente sanguínea, nos tecidos moles e no osso. Neste contexto, o uso de modelo matemático pode tornar-se uma ferramenta importante no estudo dos efeitos do fósforo no metabolismo animal, tendo em vista sua precisão nas estimativas dos dados.

O objetivo neste trabalho foi avaliar a aplicação de dois modelos matemáticos no estudo do metabolismo de fontes de fósforo para suínos.

\section{Material e Métodos}

O experimento foi desenvolvido no Laboratório de Nutrição Animal - CENA/USP, utilizando-se 21 suínos híbridos machos castrados com peso médio de $32 \mathrm{~kg}$, istribuídos em delineamento de blocos casualizados, com sete dietas e três repetições e um animal por unidade experimental, alojados em gaiolas metabólicas semelhantes às descritas por Pekas (1968).

As dietas foram constituídas de uma ração basal composta de milho, farelo de soja e óleo vegetal e suplementada com fosfato bicálcico, fosfato monobicálcio, superfosfato triplo, superfosfato simples, ácido fosfórico ou fosfato de rocha catalão como fontes de fósforo (Tabela 1).
O experimento teve duração de 14 dias: sete correspondentes à fase pré-experimental, para adaptação dos animais às gaiolas e às dietas; e sete à fase experimental, para coletas.

No primeiro dia da fase experimental, cada animal recebeu, por via endovenosa, $0,5 \mathrm{~mL}$ de uma solução radioativa com 7,4 MBq de ${ }^{32} \mathrm{P}$. A solução radioativa, preparada de acordo com Vitti et al. (2006), foi constituída de fosfato de sódio $\left(\mathrm{Na}_{2} \mathrm{H}^{32} \mathrm{PO}_{4}\right)$ livre de carregador e adquirida no Instituto de Pesquisas Energéticas e Nucleares (IPEN). Nos três últimos dias da fase pré-experimental, foram coletadas amostras de fezes para determinação do fósforo inorgânico.

Após a aplicação do material radioativo, foram coletadas diariamente amostras de sangue, fezes e urina. No final do período experimental, os suínos foram sacrificados de acordo com aprovação da Comissão de Ética em Experimentação com animais (CEEA) do Centro de Energia Nuclear da Agricultura CENA) e amostras de tecidos (ósseo, muscular esquelético, lombar, cardíaco, hepático e renal) foram coletadas.

As amostras de sangue foram centrifugadas imediatamente após a coleta a 1100 x $g$ durante 10 minutos e o plasma foi separado. Para a detecção da radioatividade, 0,5 mL de plasma foi adicionado em água deionizada em frasco de contagem e a determinação foi obtida por efeito Cerenkov (Vitti et al., 2006). Ao volume de $1 \mathrm{~mL}$ de plasma foram adicionados $9 \mathrm{~mL}$ de ácido tricloroacético a $10 \%$ para a precipitação de proteínas, e o teor de fósforo inorgânico determinado por colorimetria (Fiske \& Subbarow, 1925).

As fezes foram coletadas durante sete dias, pesadas, maceradas e homogeneizadas e, um grama colocado em cadinho de porcelana para as determinações da matéria seca (MS) $\left(100^{\circ} \mathrm{C}\right)$ e das cinzas $\left(500^{\circ} \mathrm{C}\right)$. As cinzas foram adicionadas de $10 \mathrm{~mL}$ de ácido sulfúrico $(18 \mathrm{~N})$ e o material digerido foi colocado em frascos de cintilação completando-se o volume para $20 \mathrm{~mL}$ com água destilada e determinando-se a radioatividade por efeito Cerenkov.

Cerca de $1 \mathrm{~g}$ de fezes foi digerido com $5 \mathrm{~mL}$ de ácido clorídrico concentrado e filtrado. Ao volume de $2,5 \mathrm{~mL}$ do filtrado foram adicionados $2,5 \mathrm{ml}$ de água deionizada e $2 \mathrm{~mL}$ de reagente misto (quantidades iguais de molibdato de amônia a 5\% e vanadato de amônia a 0,25\%). As leituras do P inorgânico foram feitas por colorimetria (Sarruge \& Haag, 1974).

Das amostras de urina coletadas, 0,5 mL foi diluído em 19,5 mL de água deionizada e a atividade foi determinada por efeito Cerenkov. O fósforo inorgânico foi determinado após diluição de $1 \mathrm{~mL}$ de urina em $9 \mathrm{~mL}$ de ácido tricloroacético (10\%) (Fiske \& Subbarow, 1925). 
Os dados coletados neste experimento foram utilizados para o desenvolvimento dos dois modelos baseados nos estudos de Vitti et al. (2000) e Lopes et al. (2001). No modelo proposto por Vitti et al., 2000, utilizaram-se os seguintes compartimentos anatômicos: trato digestivo, tecidos moles (cardíaco, hepático, lombar e renal) e ossos.

Na elaboração do modelo do fluxo do fósforo no metabolismo de suínos, foram medidas as variáveis de entrada no sistema [P consumido $\left(\mathrm{F}_{10}\right)$ ] e de saída [excreção fecal $\left(\mathrm{F}_{01}\right)$ e urinária $\left(\mathrm{F}_{02}\right)$ de $\left.\mathrm{P}\right]$, bem como as atividades específicas do ${ }^{32} \mathrm{P}$ nas fezes, no sangue, no osso e nos tecidos moles $\left(\mathrm{S}_{1}, \mathrm{~S}_{2}, \mathrm{~S}_{3}\right.$ e $\left.\mathrm{S}_{4}\right)$ e outras entradas, como a quantidade de fósforo no sangue, no osso e nos tecidos $\left(\mathrm{Q}_{2}, \mathrm{Q}_{3}, \mathrm{Q}_{4}\right)$, e outras saídas, representadas pelo fluxo bidirecional de fósforo entre o sangue e o trato digestivo, os ossos e os tecidos moles $\left(\mathrm{F}_{12}, \mathrm{~F}_{21}, \mathrm{~F}_{32}, \mathrm{~F}_{42}, \mathrm{~F}_{2324}, \mathrm{~F}_{24}, \mathrm{~F}_{23}\right)$ (Figura 1).

No modelo de Vitti para a distribuição de fósforo no metabolismo de suínos, os símbolos indicam: $\mathrm{F}_{\mathrm{ij}}=$ fluxo total de fósforo para o pool $\mathrm{i}$ a partir do $\mathrm{J}$, em que $\mathrm{F}_{\mathrm{i} 0}=$ fluxo de entrada no sistema e $\mathrm{F}_{0 \mathrm{j}}=$ fluxo de saída a partir do pool j; os parâmetros $\mathrm{F}_{10}, \mathrm{~F}_{01}$ e $\mathrm{F}_{02}$ indicam o fluxo que pode ser medido experimentalmente (g/dia); D = dose de ${ }^{32} \mathrm{P}$ administrada no sangue no tempo zero (cpm); Qi = quantidade total de fósforo no pool i (g); $\mathrm{q}_{\mathrm{i}}=$ quantidade de ${ }^{32} \mathrm{P}$ no pool i (cpm); $\mathrm{S}_{\mathrm{i}}=$ atividade específica do pool i $\left(=\mathrm{q}_{\mathrm{i}} / \mathrm{Q}_{\mathrm{i}}\right)(\mathrm{cpm} / \mathrm{g})$; $\mathrm{T}=$ Tempo (dias).

O princípio da conservação das massas pode ser aplicado para cada compartimento (Figura 1) para gerar as equações diferenciais, apresentadas a seguir, as quais descrevem o comportamento dinâmico do sistema. Assim, as equações diferenciais de 1 a 4 representam esse princípio para o fósforo não marcado, e as equações de 5 a 8 para o fósforo marcado.

$$
\begin{array}{lr}
\mathrm{dQ}_{1} / \mathrm{dt}=\mathrm{F}_{10}+\mathrm{F}_{12}-\mathrm{F}_{01}-\mathrm{F}_{21} & \text { (equação 1) } \\
\mathrm{dQ}_{2} / \mathrm{dt}=\mathrm{F}_{21}+\mathrm{F}_{23}+\mathrm{F}_{24}-\mathrm{F}_{02}-\mathrm{F}_{12}-\mathrm{F}_{32}-\mathrm{F}_{42} & \text { (equação 2) } \\
\mathrm{dQ}_{3} / \mathrm{dt}=\mathrm{F}_{32}-\mathrm{F}_{23} & \text { (equação 3) } \\
\mathrm{dQ}_{4} / \mathrm{dt}=\mathrm{F}_{42}-\mathrm{F}_{24} & \text { (equação 4) } \\
\mathrm{dq}_{1} / \mathrm{dt}=\mathrm{S}_{2} \mathrm{~F}_{12}-\mathrm{S}_{1}\left(\mathrm{~F}_{01}-\mathrm{F}_{21}\right) & (\text { equação 5) } \\
\mathrm{dq}_{2} / \mathrm{dt}=\mathrm{S}_{1} \mathrm{~F}_{21}+\mathrm{S}_{3} \mathrm{~F}_{23}+\mathrm{S}_{4} \mathrm{~F}_{24}-\mathrm{S}_{2}\left(\mathrm{~F}_{02}+\mathrm{F}_{12}+\mathrm{F}_{32}+\mathrm{F}_{42}\right)
\end{array}
$$

\begin{tabular}{|c|c|c|c|c|c|c|c|}
\hline \multirow[t]{2}{*}{ Ingrediente } & \multicolumn{7}{|c|}{ Dieta experimental } \\
\hline & Controle & $\begin{array}{l}\text { Fosfato } \\
\text { bicálcico }\end{array}$ & $\begin{array}{c}\text { Fosfato } \\
\text { monobicálcico }\end{array}$ & $\begin{array}{l}\text { Superfosfato } \\
\text { triplo }\end{array}$ & $\begin{array}{l}\text { Superfosfato } \\
\text { simples }\end{array}$ & $\begin{array}{c}\text { Ácido } \\
\text { fosfórico }\end{array}$ & $\begin{array}{l}\text { Fosfato de } \\
\text { rocha }\end{array}$ \\
\hline Farelo de soja & 26,30 & 26,30 & 26,30 & 26,30 & 26,30 & 26,30 & 26,30 \\
\hline Milho & 68,00 & 68,00 & 68,00 & 68,00 & 68,00 & 68,00 & 68,00 \\
\hline L-lisina & 0,10 & 0,10 & 0,10 & 0,10 & 0,10 & 0,10 & 0,10 \\
\hline Óleo de soja & 1,65 & 1,65 & 1,65 & 1,65 & 1,65 & 1,65 & 1,65 \\
\hline Sal & 0,30 & 0,30 & 0,30 & 0,30 & 0,30 & 0,30 & 0,30 \\
\hline Mistura mineral ${ }^{1}$ & 0,05 & 0,05 & 0,05 & 0,05 & 0,05 & 0,05 & 0,05 \\
\hline Mistura vitamínica ${ }^{1}$ & 0,10 & 0,10 & 0,10 & 0,10 & 0,10 & 0,10 & 0,10 \\
\hline Areia lavada & 1,78 & 1,23 & 1,15 & 1,06 & 0,0 & 0,70 & 1,62 \\
\hline Calcário & 1,72 & 0,99 & 1,17 & 1,28 & 0,36 & 1,78 & 0,00 \\
\hline Fosfato bicálcico ${ }^{3}$ & & 1,28 & & & & & \\
\hline Fosfato monobicálcico ${ }^{3}$ & & & 1,18 & & & & \\
\hline Superfosfato triplo ${ }^{3}$ & & & & 1,160 & & & \\
\hline Superfosfato simples ${ }^{3}$ & & & & & 3,14 & & \\
\hline Ácido fosfórico ${ }^{3}$ & & & & & & 1,02 & \\
\hline Fosfato de rocha catalão ${ }^{3}$ & & & & & & & 1,88 \\
\hline \multicolumn{8}{|l|}{ Composição calculada } \\
\hline Energia digestível kcal/kg & 3400 & 3400 & 3400 & 34000 & 3400 & 34000 & 3400 \\
\hline Proteína bruta (\%) & 17,90 & 17,90 & 17,90 & 17,90 & 17,90 & 17,90 & 17,90 \\
\hline Fósforo total $(\%)$ & 0,321 & 0,560 & 0,560 & 0,560 & 0,560 & 0,560 & 0,560 \\
\hline Cálcio (\%) & 0,780 & 0,780 & 0,780 & 0,780 & 0,780 & 0,780 & 0,780 \\
\hline Sódio (\%) & 0,157 & 0,157 & 0,157 & 0,157 & 0,157 & 0,157 & 0,157 \\
\hline Lisina total (\%) & 0,980 & 0,980 & 0,980 & 0,980 & 0,980 & 0,980 & 0,980 \\
\hline Metionina (\%) & 0,287 & 0,287 & 0,287 & 0,287 & 0,287 & 0,287 & 0,287 \\
\hline Metionina + cistina (\%) & 0,586 & 0,586 & 0,586 & 0,586 & 0,586 & 0,586 & 0,586 \\
\hline Treonina (\%) & 0,693 & 0,693 & 0,693 & 0,693 & 0,693 & 0,693 & 0,693 \\
\hline Triptofano (\%) & 0,212 & 0,212 & 0,212 & 0,212 & 0,212 & 0,212 & 0,212 \\
\hline
\end{tabular}

(equação 6)

Tabela 1 - Composição das dietas fornecidas na fase de crescimento 
$\mathrm{dq}_{3} / \mathrm{dt}=\mathrm{S}_{2} \mathrm{~F}_{32}-\mathrm{S}_{3} \mathrm{~F}_{23}$

(equação 7)

$\mathrm{dq}_{4} / \mathrm{dt}=\mathrm{S}_{2} \mathrm{~F}_{42}-\mathrm{S}_{4} \mathrm{~F}_{24}$

(equação 8)

Quando se considera o coeficiente de diferencial de $\mathrm{S}_{3}$ no tempo, tem-se:

$\mathrm{dS}_{3} / \mathrm{dt}=\mathrm{d}\left(\mathrm{q}_{3} / \mathrm{Q}_{3-}{ }^{1}\right) / \mathrm{dt}=\left[\mathrm{dq}_{3} / \mathrm{dt}-\left(\mathrm{q}_{3} / \mathrm{Q}_{3}\right) \mathrm{dQ}_{3} / \mathrm{dt}\right] / \mathrm{Q}_{3}$

(equação 9)

Reorganizando o sistema de equações, obtém-se:

$\mathrm{dq}_{3} / \mathrm{dt}=\mathrm{Q}_{3} \mathrm{dS}_{3} / \mathrm{dt}+\mathrm{S}_{3} \mathrm{dQ}_{3} / \mathrm{dt}$

(equação 10)

$\mathrm{E}$, quando se utilizam as equações 3 e 7 para substituir $\mathrm{dQ}_{3} / \mathrm{dt}$ e $\mathrm{dq}_{3} / \mathrm{dt}$, respectivamente, aproximando-se $\mathrm{dS}_{3} / \mathrm{dt}$ por $\left[\mathrm{S}_{3}(\mathrm{t}=\mathrm{t})-\mathrm{S}_{3}(\mathrm{t}=0)\right] /$ [ $\left.\mathrm{t}-0\right]$, a equação 10 torna-se: $\mathrm{S}_{3} / \mathrm{t}=\left(\mathrm{S}_{2}-\mathrm{S}_{3}\right) \mathrm{F}_{32} / \mathrm{Q}_{3}$

(equação 11)

$\mathrm{Como}_{3}(\mathrm{t}=0)$ é zero, e considerando-se similar, logo, ao aplicar para $\mathrm{ds}_{4} / \mathrm{dt}$, tem-se:

$\mathrm{S}_{4} / \mathrm{t}=\left(\mathrm{S}_{2}-\mathrm{S}_{4}\right) \mathrm{F}_{42} / \mathrm{Q}_{4}$

(equação 12)

Oito dias depois da injeção do material radioativo, pressupõe-se que o pool 1 (lúmen do intestino) encontra-se em equilíbrio (i.e., ambos $\mathrm{dQ}_{1} / \mathrm{dt}$ são zero) e que o compartimento 2 (sangue) acha-se em estado não isotópico estável (i.e., $\mathrm{dQ}_{2}$ /dt é zero). Assim, as equações 1, 2, 5, 11 e 12 se transformam:

$\mathrm{F}_{10}+\mathrm{F}_{12}-\mathrm{F}_{01}-\mathrm{F}_{21}=0$

(equação 13)

$\mathrm{F}_{21}+\mathrm{F}_{23}+\mathrm{F}_{24}-\mathrm{F}_{02}-\mathrm{F}_{12}-\mathrm{F}_{32}-\mathrm{F}_{42}=0$

(equação 14)

$\mathrm{S}_{2} \mathrm{~F}_{12}-\mathrm{S}_{1}\left(\mathrm{~F}_{01}+\mathrm{F}_{02}\right)=0$

(equação 15)

$\left(\mathrm{S}_{2}-\mathrm{S}_{3}\right) \mathrm{F}_{32} / \mathrm{Q}_{3}=\mathrm{S}_{3} / 8$

(equação 16)

$\left(\mathrm{S}_{2}-\mathrm{S}_{4}\right) \mathrm{F}_{42} / \mathrm{Q}_{4}=\mathrm{S}_{4} / 8$

(equação 17)

Por meio da manipulação algébrica das equações 13 e

17, tem-se o resultado:

$\mathrm{F}_{12}=\mathrm{S}_{1} \mathrm{~F}_{10} /\left(\mathrm{S}_{2}-\mathrm{S}_{1}\right)$

(equação 18)

$\mathrm{F}_{21}=\mathrm{F}_{10}+\mathrm{F}_{12}-\mathrm{F}_{01}$

(equação 19)

$\mathrm{F}_{32}=\mathrm{S}_{3} \mathrm{Q}_{3} /\left[8\left(\mathrm{~S}_{2}-\mathrm{S}_{3}\right)\right]$

(equação 20)

$\mathrm{F}_{42}=\mathrm{S}_{4} \mathrm{Q}_{4} /\left[8\left(\mathrm{~S}_{2}-\mathrm{S}_{4}\right)\right]$

(equação 21)

$\left|\mathrm{F}_{23}+\mathrm{F}_{24}\right|=\mathrm{F}_{02}+\mathrm{F}_{12}+\mathrm{F}_{32}+\mathrm{F}_{42}-\mathrm{F}_{21}$

(equação 22)

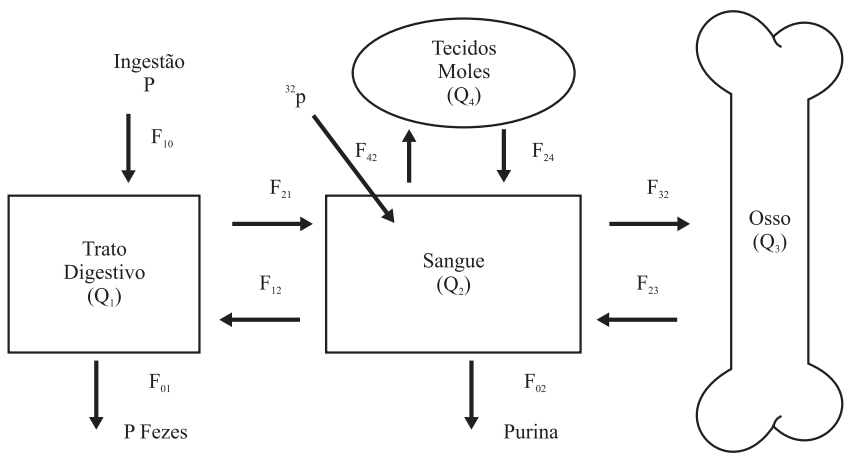

Figura 1 - Modelo de Vitti et al. (2000) para distribuição de fósforo no metabolismo de suínos. em que o $\left|F_{23}+F_{24}\right|$ indica o fluxo experimentalmente medido. O fluxo combinado $\left|\mathrm{F}_{23}+\mathrm{F}_{24}\right|$ denota a soma do fluxo de saída do pool 3 e do pool 4 (equação 23) e pode ser separado pela combinação desses dois pools. O S* denota a atividade específica desse pool combinado, calculado de acordo com a equação 24 :

$\left|\mathrm{F}_{23}+\mathrm{F}_{24}\right|=\mathrm{F}_{23}+\mathrm{F}_{24}$

(equação 23)

$\mathrm{S}^{*}=\left(\mathrm{S}_{3} \mathrm{Q}_{3}+\mathrm{S}_{4} \mathrm{Q}_{4}\right) /\left(\mathrm{Q}_{3}+\mathrm{Q}_{4}\right)$

(equação 24)

O fluxo de saída do marcador a partir do pool combinado constitui na soma do fluxo de saída do marcador dos pools 3 e 4 .

$\mathrm{S}^{*} \times\left|\mathrm{F}_{23}+\mathrm{F}_{24}\right|=\mathrm{S}_{3} \mathrm{~F}_{23}+\mathrm{S}_{4} \mathrm{~F}_{24}$

(equação 25)

Por meio de manipulação algébrica das equações 23 e 25, obtém-se:

$\mathrm{F}_{24}=\left(\mathrm{S}^{*}-\mathrm{S}_{3}\right) \times\left|\mathrm{F}_{23}+\mathrm{F}_{24}\right| /\left(\mathrm{S}_{4}-\mathrm{S}^{*}\right)$

$\mathrm{F}_{23}=\left|\mathrm{F}_{23}+\mathrm{F}_{24}\right|-\mathrm{F}_{24}$

(equação 26)

(equação 27)

O modelo é aplicado usando as equações 18 a 22, 24 e 26 a 27 para computar os fluxos desconhecidos.

O modelo de Lopes et al. (2001) foi constituído pelos compartimentos $\mathrm{C}_{1}$ trato gastrintestinal, $\mathrm{C}_{2}$ plasma, $\mathrm{C}_{3}$ ossos e $\mathrm{C}_{4}$ tecidos moles, sendo o fígado, coração, rim e músculos estudados em conjunto, de acordo com a proporção dos seus pesos (Figura 2).

As variáveis relacionadas ao metabolismo de fósforo no modelo de Lopes et al. (2001) foram: VI = quantidade de fósforo ingerido; $\mathrm{VF}$ = perda total de fósforo nas fezes; $\mathrm{Vf}$ = fósforo endógeno fecal; VFD = fósforo de origem dietética nas fezes; VU = perda de fósforo na urina; VIT = fósforo de origem endógena que chega ao trato gastrointestinal (TGI); Vaf = fósforo endógeno reabsorvido no TGI; $\mathrm{VaT}$ = total de fósforo absorvido; $\mathrm{VeD} 1$ = fósforo

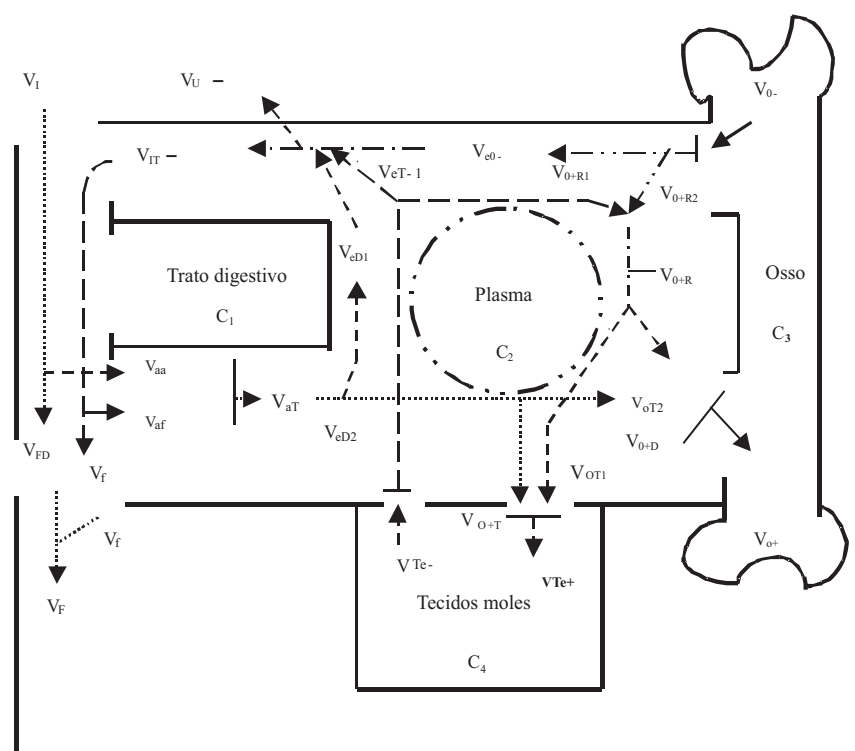

Figura 2 - Modelo de fluxo do fósforo no metabolismo e suínos. 
do VaT que retorna ao TGI; VeD2 = fósforo do VaT distribuído para os ossos e tecidos moles; $\mathrm{VO}+\mathrm{T}$ = fósforo do $\mathrm{VaT}$ incorporado nos tecidos moles; $\mathrm{VO}+\mathrm{D}=$ fósforo do $\mathrm{VaT}$ incorporado nos ossos; $\mathrm{VO}+=$ fósforo incorporado nos ossos; $\mathrm{VO}$ - = fósforo reabsorvido dos ossos; $\mathrm{VeO}$ - = fósforo reabsorvido dos ossos que retorna ao TGI; $\mathrm{VO}+\mathrm{R} 1$ = fósforo dos tecidos moles reciclado para os ossos e tecidos moles; $\mathrm{VO}+\mathrm{R} 2=\mathrm{P}$ dos ossos reciclado para os ossos e tecidos moles; $\mathrm{VO}+\mathrm{R}=$ total de fósforo reciclado para os ossos e tecidos moles; $\mathrm{VOT} 1$ = fósforo do $\mathrm{VO}+\mathrm{R}$ reincorporado nos tecidos moles; $\mathrm{VOT} 2$ = fósforo do $\mathrm{VO}+\mathrm{R}$ reincorporado nos ossos; $\mathrm{VTe}+$ = fósforo incorporado nos tecidos moles; $\mathrm{VTe}$ - = fósforo reabsorvido dos tecidos moles; VeT- = fósforo reabsorvido dos tecidos moles que retorna ao TGI.

Os parâmetros foram estimados por meio de equações envolvendo as seguintes variáveis: $\mathrm{V}_{\mathrm{I}}, \mathrm{V}_{\mathrm{F}}, \mathrm{V}_{\mathrm{FD}}, \mathrm{V}_{\mathrm{f}}, \mathrm{V}_{\mathrm{U}}, \mathrm{V}_{\mathrm{aa}}$, $\mathrm{V}_{\mathrm{af}}, \mathrm{V}_{\mathrm{IT}}$ e $\mathrm{V}_{\mathrm{aT}}$, definidas segundo Fernandez (1995) (equações 1 a 6 ).

O valor do fósforo proveniente do total absorvido $\left(\mathrm{V}_{\mathrm{aT}}\right)$ e que retorna ao trato gastrintestinal $\left(\mathrm{V}_{\mathrm{eD} 1}\right)$ foi calculado pela diferença entre o fósforo do $\mathrm{V}_{\mathrm{aT}}$ e o fósforo incorporado em $\mathrm{C}_{3} \mathrm{e}_{4}$ após subtrair-se o fósforo reciclado que retorna a esses compartimentos. O restante do total absorvido $\left(\mathrm{V}_{\mathrm{eD} 2}\right)$ foi distribuído proporcionalmente entre $\mathrm{C}_{3}$ e $\mathrm{C}_{4}\left(\mathrm{~V}_{0+\mathrm{T}}\right.$ e $\left.\mathrm{V}_{0+\mathrm{D}}\right)$, de acordo com as equações $7-10$. $\mathrm{V}_{\mathrm{FD}}=\mathrm{VF}-\mathrm{Vf}$ (equação 1 ) $\mathrm{V}_{\text {aa }}=\mathrm{V}_{\mathrm{I}}-\mathrm{V}_{\mathrm{FD}}$ (equação 2) $\mathrm{V}_{\mathrm{IT}}=\mathrm{V}_{\mathrm{f}} /\left[1-\left(\mathrm{V}_{\mathrm{aa}} / \mathrm{V}_{\mathrm{I}}\right)\right]$ (equação 3 ) $\mathrm{V}_{\mathrm{af}}=\mathrm{V}_{\mathrm{IT}}-\mathrm{V}_{\mathrm{f}}$ (equação 4 ) $\mathrm{V}_{\mathrm{aT}}=\mathrm{V}_{\mathrm{aa}}+\mathrm{V}_{\mathrm{af}}$ (equação 5 ) $\mathrm{B}_{\mathrm{a}}=\mathrm{V}_{\mathrm{I}}-\mathrm{V}_{\mathrm{F}}-\mathrm{V}_{\mathrm{U}}$ (equação 6 ) $\mathrm{V}_{\mathrm{eD} 1}=\mathrm{V}_{\mathrm{aT}}-\left(\mathrm{V}_{\mathrm{O}^{+}}-\mathrm{V}_{\mathrm{oR} 2}\right)-\left(\mathrm{V}_{\mathrm{Te}^{+}}-\mathrm{V}_{\mathrm{o}^{+\mathrm{R} 1}}\right)$ (equação 7$)$ $\mathrm{V}_{\mathrm{eD} 2}=\mathrm{V}_{\mathrm{aT}}-\mathrm{V}_{\mathrm{eD} 1} \quad$ (equação 8) $\mathrm{V}_{\mathrm{O}+\mathrm{D}}=\frac{\left[\mathrm{V}_{\mathrm{o}^{+}} \times\left(\mathrm{V}_{\mathrm{aT}} \times \% \text { P incorporado no osso }\right)\right]}{\left[\mathrm{V}_{\mathrm{O}^{-}} \times\left(\mathrm{V}_{\mathrm{aT}} \times \% \text { P incorporado no osso }\right)\right]}$ (equação 9) $\mathrm{V}_{\mathrm{o}+\mathrm{T}}=\frac{\left[\mathrm{V}_{\mathrm{Te}^{+}} \times\left(\mathrm{V}_{\mathrm{aT}} \times \% \text { P incorporado nos tecidos }\right)\right]}{\left[\mathrm{V}_{\mathrm{Te}-} \times\left(\mathrm{V}_{\mathrm{aT}} \times \% \text { P incorporado nos tecidos }\right)\right]}$ (equação 10)

Na determinação do fósforo incorporado no osso $\left(\mathrm{V}_{0_{+}}\right)$e nos tecidos moles, hepático, cardíaco, renal, muscular esquelético e lombar $\left(\mathrm{V}_{\mathrm{Te}+}\right)$, considerou-se a concentração desse mineral (mg/gMS). O percentual de ${ }^{32} \mathrm{P}$ [(cpm amostra/ g de MS)/(dose total injetada)]/[(mgP/g MS)] e a atividade específica dos tecidos [(\% da atividade injetada de $\left.{ }^{32} \mathrm{P}\right) /$ (mgP/g MS do tecido)] foram calculados de acordo com Lopes (1998).
Para o ${ }^{32} \mathrm{P}$ retido nos tecidos moles, foi considerada a média dos valores de cada tecido estudado. A quantidade de MS de cada tecido foi determinada pelo produto entre o percentual de MS obtida e o peso dos tecidos, definidos segundo dados de pesquisa de Davidson (1966) e Lopes (1988). Foi utilizado ainda neste processo o decaimento físico (T1/2) e biológico (Tb) do ${ }^{32} \mathrm{P}$, fundamentado na International Atomic Energy Agency (1979), uma vez que os animais foram sacrificados uma semana após a aplicação do ${ }^{32} \mathrm{P}$ e a contagem do material radioativo foi feita oito dias após o abate.

$\mathrm{O}$ valor do ${ }^{32} \mathrm{P}$ retido (mg/gMS) foi determinado pelo produto do percentual de ${ }^{32} \mathrm{P}$ retido e a concentração total de fósforo nos compartimentos $\mathrm{C}_{1}$ e $\mathrm{C}_{3}$ (mg/gMS).

Após a correção do decaimento do ${ }^{32} \mathrm{P}$ para a data de sua introdução na corrente sanguínea, calculou-se o fósforo incorporado (mg/g MS) nos compartimentos $3 \mathrm{e}$ 4 pela relação entre o valor de ${ }^{32} \mathrm{P}$ corrigido e o da atividade específica em cada compartimento envolvido, e o fósforo total incorporado (mg) nos compartimentos $\mathrm{C}_{3}$ e $\mathrm{C}_{4}$ foi obtido pelo produto entre a MS (g) do compartimento e o fósforo incorporado (mg). Os valores de $\mathrm{V}_{0^{+}} \mathrm{e} \mathrm{V}_{\mathrm{Te}^{+}}(\mathrm{mgP} / \mathrm{kgPV}$. dia) foram obtidos pela relação entre o fósforo total incorporado no compartimento e o peso do animal (g). As equações 11 a 24 caracterizam essas determinações.

${ }^{32} \mathrm{P}$ retido no osso $\left(\mathrm{mg}{ }^{32} \mathrm{P} / \mathrm{g} \mathrm{MS}\right)$ = fósforo inorgânico $(\mathrm{mg} \mathrm{P} / \mathrm{gMS}) \times{ }^{32} \mathrm{P}$ retido $(\%)$

(equação 11)

$\mathrm{P}$ total incorporado osso $(\mathrm{mg} \mathrm{P})$ = fósforo total osso $(\mathrm{mg} \mathrm{P} / \mathrm{g}$ MS) $\times$ MS osso $(\mathrm{g}$ MS)

(equação 12)

$\mathrm{P}$ total incorporado osso (mg P)

$\mathrm{Vo}+(\mathrm{mg} \mathrm{P} / \mathrm{kg}$ peso animal $)=-\times 1000$

Peso total do suíno (kg)

(equação 13)

Peso total tecidos $(\mathrm{g})=$ peso suíno $(\mathrm{g}) \times \%$ peso tecidos

(equação 14)

MS tecidos $(\mathrm{g})=$ peso total tecidos $(\mathrm{g}) \times \%$ MS tecidos (equação 15)

${ }^{32} \mathrm{P}$ retido fígado $\left(\mathrm{mg}{ }^{32} \mathrm{P} / \mathrm{g}\right.$ MS $)$ = fósforo inorgânico $(\mathrm{mg} \mathrm{P} / \mathrm{g} \mathrm{MS}) \times \%{ }^{32} \mathrm{P}$ retido

(equação 16)

${ }^{32} \mathrm{P}$ retido coração $\left(\mathrm{mg}{ }^{32} \mathrm{P} / \mathrm{g} \mathrm{MS}\right)$ = fósforo inorgânico $(\mathrm{mg} \mathrm{P} / \mathrm{g} \mathrm{MS}) \times \%{ }^{32} \mathrm{P}$ retido (equação 17)

${ }^{32} \mathrm{P}$ retido $\operatorname{rim}\left(\mathrm{mg}{ }^{32} \mathrm{P} / \mathrm{g} \mathrm{MS}\right)=$ fósforo inorgânico $(\mathrm{mg} \mathrm{P} / \mathrm{g} \mathrm{MS}) \times \%{ }^{32} \mathrm{P}$ retido (equação 18)

${ }^{32} \mathrm{P}$ retido músculo $\left(\mathrm{mg}{ }^{32} \mathrm{P} / \mathrm{g}\right.$ MS) = fósforo inorgânico $(\mathrm{mg} \mathrm{P} / \mathrm{g}$ MS $) \times \%{ }^{32} \mathrm{P}$ retido (equação 19)

$\mathrm{P}$ total incorporado tecido $(\mathrm{mg} \mathrm{P})=$ fósforo total tecido $(\mathrm{mg}$ P/g MS) $\times$ MS tecido (g)

(equação 20) 
P total incorporado tecido (mg P)

$\mathrm{V}_{\mathrm{Te}^{+}}(\mathrm{mg} \mathrm{P} / \mathrm{kg}$ peso animal $)=\longrightarrow 1000$

Peso do suíno (kg)

(equação 21)

$\mathrm{T} 1 / 2=0,693$ /constante de decaimento (K) (equação 22)

Atividade do material radioativo no tempo “ $\mathrm{t}$ ” $(\mathrm{A})=$ Atividade inicial $\left(A_{0}\right) \times e^{-k t}$

(equação 23)

$\mathrm{Tb}=0,693 \mathrm{t} / \ln \left(\mathrm{A}_{\mathrm{o}} / \mathrm{A}\right)$

(equação 24)

O fósforo reabsorvido nos compartimentos $\mathrm{C}_{3}\left(\mathrm{~V}_{0_{-}}\right) \mathrm{e}$ $\mathrm{C}_{4}\left(\mathrm{~V}_{\mathrm{Te}}\right)$ foi calculado deduzindo-se, respectivamente, de $\mathrm{V}_{0_{+}}$e de $\mathrm{V}_{\mathrm{Te}+} \mathrm{o}$ valor do balanço de fósforo multiplicado pela proporção incorporada no compartimento em estudo (equações 25 e 26).

Para determinação do fósforo reciclado que retorna aos compartimentos $\mathrm{C}_{3}$ e $\mathrm{C}_{4}\left(\mathrm{~V}_{0+\mathrm{R} 1}\right.$ e $\left.\mathrm{V}_{0+\mathrm{R} 2}\right)$ e ao trato gastrintestinal $\left(\mathrm{V}_{\mathrm{eo}}-\mathrm{V}_{\mathrm{eT}}\right)$, o cálculo foi realizado com base nas equações 27 a 30, segundo Fernandez (1995) e Lopes et al. (2001), adaptando-se ao modelo a proporção incorporada em cada um dos dois compartimentos, visto que neste trabalho o osso foi o único compartimento que mobilizou fósforo.

O valor do fósforo proveniente do osso e tecidos moles reciclado para os tecidos moles $\left(\mathrm{V}_{0+\mathrm{R}}\right)$, o do fósforo do $\mathrm{V}_{0+\mathrm{R}}$ reincorporado nos tecidos moles $\left(\mathrm{V}_{\mathrm{TO} 1}\right)$ e o fósforo do $\mathrm{V}_{\mathrm{O}+\mathrm{R}}$ reincorporado nos ossos $\left(\mathrm{V}_{\mathrm{T} 02}\right)$ foram determinados de acordo com as equações 31 a 33.

$\mathrm{V}_{\mathrm{O}_{-}}=\mathrm{V}_{0^{+}}-(\mathrm{BA} \times \% \mathrm{P}$ incorporado no osso $)$

(equação 25)

$$
\mathrm{V}_{\mathrm{TE}-}=\mathrm{V}_{\mathrm{TE}+}-(\mathrm{BA} \times \% \mathrm{P} \text { incorporado tecido })
$$

$$
\mathrm{V}_{0+\mathrm{R} 1}=\frac{\mathrm{V}_{\mathrm{Te}+} \times \mathrm{V}_{\mathrm{Te}-}}{\left[\mathrm{V}_{\mathrm{TE}+}\left(\mathrm{V}_{\mathrm{AT}} \times \% \mathrm{P} \text { incorporado tecido }\right)\right]}
$$

(equação 26)

$$
\mathrm{V}_{0+\mathrm{R} 2}=\frac{\mathrm{V}_{\mathrm{O}^{+}} \times \mathrm{V}_{\mathrm{o}-}}{\mathrm{V}_{\mathrm{O}^{-}}+\left(\mathrm{V}_{\mathrm{aT}} \times \% \text { P incorporado osso }\right)}
$$

(equação 27)

$$
\begin{aligned}
\mathrm{V}_{\mathrm{eo}-} & =\mathrm{V}_{\mathrm{o}-}-\mathrm{V}_{\mathrm{o}+\mathrm{R} 2} \\
\mathrm{~V}_{\mathrm{eT}-} & =\mathrm{V}_{\mathrm{Te}-}-\mathrm{V}_{\mathrm{oR} 1} \\
\mathrm{~V}_{\mathrm{O}+\mathrm{R}} & =\mathrm{V}_{\mathrm{O}+\mathrm{R} 1}+\mathrm{V}_{\mathrm{O}+\mathrm{R} 2} \\
\mathrm{~V}_{\mathrm{To} 1} & =\mathrm{V}_{\mathrm{O}+\mathrm{R}}-\left(\mathrm{V}_{\mathrm{O}+}-\mathrm{V}_{\mathrm{O}+\mathrm{D}}\right) \\
\mathrm{V}_{\mathrm{To} 2} & =\mathrm{V}_{\mathrm{O}+\mathrm{R}}-\mathrm{V}_{\mathrm{To} 1}
\end{aligned}
$$

A biodisponibilidade das dietas foi determinada pela relação entre o fósforo absorvido de origem alimentar $\left(\mathrm{V}_{\mathrm{aa}}\right)$ e o fósforo consumido $\left(\mathrm{V}_{\mathrm{I}}\right)$ (Teixeira et al., 2004).

A variáveis do modelo foram submetidas à analise da variância e ao teste Duncan seguindo os procedimentos do SAS (2000).

\begin{tabular}{|c|c|c|c|c|c|c|c|}
\hline & \multicolumn{7}{|c|}{ Fonte de fósforo } \\
\hline & Controle & $\begin{array}{l}\text { Fosfato } \\
\text { bicálcico }\end{array}$ & $\begin{array}{c}\text { Fosfato } \\
\text { monobicálcico }\end{array}$ & $\begin{array}{c}\text { Superfosfato } \\
\text { triplo }\end{array}$ & $\begin{array}{l}\text { Superfosfato } \\
\text { simples }\end{array}$ & $\begin{array}{c}\text { Ácido } \\
\text { fosfórico }\end{array}$ & $\begin{array}{l}\text { Fosfato } \\
\text { de rocha } \\
\text { catalão }\end{array}$ \\
\hline \multicolumn{8}{|l|}{ Fluxo (g/dia) } \\
\hline Consumo & $3.54 b$ & $7,09 a$ & $7.25 a$ & $7.21 \mathrm{a}$ & $6.87 \mathrm{a}$ & $7.17 \mathrm{a}$ & $7.17 \mathrm{a}$ \\
\hline Fezes & $2.12 \mathrm{c}$ & $2.43 \mathrm{bc}$ & 3.19abc & $4.36 \mathrm{a}$ & $2.62 \mathrm{bc}$ & $2.35 \mathrm{bc}$ & $3.52 \mathrm{ab}$ \\
\hline \multicolumn{7}{|c|}{ Modelo de Vitti et al. (2000)* } & $0,12 \mathrm{a}$ \\
\hline Trato gastrointestinal para plasma & $1,85 b$ & $5,02 \mathrm{a}$ & $5,16 a$ & 3,96a & 4,93a & $5,94 a$ & $4,08 \mathrm{a}$ \\
\hline Plasma para trato gastrointestinal & $0,43 \mathrm{ab}$ & $0,36 b$ & $1,10 \mathrm{a}$ & $1,10 \mathrm{a}$ & $0,68 \mathrm{ab}$ & $1,12 \mathrm{a}$ & $0,43 \mathrm{ab}$ \\
\hline Plasma para o osso & $6.83 a b$ & $6,12 \mathrm{ab}$ & $9,45 \mathrm{ab}$ & $6,74 \mathrm{ab}$ & 4,43b & $7,40 \mathrm{ab}$ & $11,68 a$ \\
\hline Osso para plasma & $6,71 \mathrm{ab}$ & $2,67 \mathrm{~b}$ & $6,49 \mathrm{ab}$ & $5,09 a b$ & $1,80 \mathrm{~b}$ & 4,47ab & $8,97 a$ \\
\hline Plasma para tecidos moles & $2,45 a$ & $1,51 \mathrm{a}$ & $1,73 a$ & $1,95 \mathrm{a}$ & $1,73 a$ & $2,41 \mathrm{a}$ & $2,39 a$ \\
\hline \multicolumn{8}{|c|}{ Modelo de Lopes et al. (2001)* } \\
\hline Trato gastrointestinal para plasma & $2,01 b$ & $5,66 a$ & $4,78 a$ & $3,96 a b$ & $4,94 a$ & 5,93a & $3,92 \mathrm{ab}$ \\
\hline Plasma para trato gastrointestinal & $0,59 \mathrm{ab}$ & $0,99 \mathrm{ab}$ & $0,72 \mathrm{ab}$ & $1,10 \mathrm{a}$ & $0,68 \mathrm{ab}$ & $1,11 \mathrm{a}$ & $0,28 b$ \\
\hline Plasma para o osso & $3,18 a$ & $3,90 \mathrm{ab}$ & $4,16 a$ & $4,29 a$ & $4,06 a$ & $4,20 a$ & $3,98 a$ \\
\hline Osso para plasma & $2,15 \mathrm{a}$ & $0,11 \mathrm{c}$ & $0,98 \mathrm{abc}$ & $2,09 \mathrm{ab}$ & $0,69 \mathrm{bc}$ & $0,61 \mathrm{a}$ & $0,98 \mathrm{abc}$ \\
\hline Plasma para tecidos moles & $0,86 a$ & $0,80 \mathrm{a}$ & $1,01 \mathrm{a}$ & $1.013 \mathrm{a}$ & $0,81 \mathrm{a}$ & $1,02 \mathrm{a}$ & $0,82 \mathrm{a}$ \\
\hline Tecidos moles para plasma & $0,58 a$ & $0,01 \mathrm{c}$ & $0,24 \mathrm{abc}$ & $0,53 a b$ & $0,14 \mathrm{c}$ & $0,14 \mathrm{c}$ & $0,20 \mathrm{bc}$ \\
\hline
\end{tabular}

\section{Resultados e Discussão}

Os animais que consumiram a dieta controle ingeriram menores quantidades de ração quando comparados com os animais que consumiram dietas contendo níveis crescentes de fitase, que apresentaram consumos similares $(\mathrm{P}>0,05)$ (Tabelas 2 e 3). Esses valores estão de acordo com o

Tabela 2 - Fluxo do fósforo no metabolismo de suínos, segundo os modelos de Vitti et al. (2000) e Lopes et al. (2001)

*Médias seguidas por letras na linha diferem pelo teste Duncan. 
esperado, pois, em ensaio de metabolismo o consumo é padronizado. Além disso, a dieta controle não continha fonte suplementar de fósforo.

Entre as fontes de fósforo estudadas, o superfosfato simples, o ácido fosfórico e o fosfato bicálcico resultaram nos menores $(\mathrm{P}>0,05)$ valores de fósforo excretado nas fezes. A limitação do uso do superfosfato simples se restringe à sua composição química, pois contém baixo teor de fósforo (8,6\%) e alto teor em cálcio (21,5\%) (Rostagno, 2005). Além disso, o superfosfato simples continha alto teor em enxofre (12\%), o que contribui para o aumento do odor dos dejetos.

Dependendo da origem da rocha, o superfosfato triplo e o simples podem conter níveis elevados de flúor e de metais pesados (Teixeira et al., 2005). Entretanto, essas duas fontes de fósforo possuem alta solubilidade em água e em ácido cítrico, devido à predominância do monocálcico. Segundo Jongbloed et al. (1990), a presença fosfatos monovalentes aumenta a digestibilidade do fósforo.

A poluição por dejetos de suínos é cerca de três vezes mais alta que a do homem. Em termos comparativos, enquanto a $\mathrm{DBO}_{5}$ (demanda bioquímica por oxigênio) per capita de um suíno com 85 kg de peso vivo varia de 189 a 208 g/animal/dia, a humana é de 45 a 75 g/pessoa/dia (Perdomo \& Lima, 1998). Isto torna obrigatório o cuidado na escolha dos melhores ingredientes para formulação das dietas. Neste experimento, 79 a $46 \%$ do fósforo consumido foi excretado nas fezes e na urina nos grupo controle e naqueles que receberam as fontes de fósforo, respectivamente.

No fluxo de fósforo do trato gastrintestinal para o plasma, os valores foram similares para as dietas suplementadas com as fontes de fósforo e foram menores para a dieta controle. Segundo Breves \& Schroder (1991), os animais monogástricos absorvem o fósforo no intestino delgado de duas maneiras: por um mecanismo ativo saturável e um passivo não-saturável, de modo que, em níveis altos de consumo de fósforo, ocorre também absorção passiva, provocada pela pressão do fósforo nos sítios de absorção (Moreira et al., 2004).

Neste experimento $45 \%$ do fósforo consumido da dieta controle foi absorvido, seguidos de 75, 68, 55, 72, 82 e 56\% para fosfato bicálcico, fosfato monobicálcico, superfosfato triplo, superfosfato simples, ácido fosfórico e fosfato de rocha catalão, respectivamente, valores similares aos obtidos por Teixeira et al. (2004a) em experimentos sobre biodisponibilidade.

O fluxo de fósforo do plasma para o trato gastrintestinal representa a perda obrigatória provocada pelo turnover celular e pela excreção de fluidos no sistema digestivo de suínos. Os animais que consumiram dietas suplementadas com as fontes de fósforo excretaram $16 \%$ do fósforo absorvido, entretanto, aqueles que se alimentaram com a dieta controle excretaram 25\% do fósforo absorvido, comprovando que a deficiência de fósforo na dieta influencia as perdas endógenas.

O nível de fósforo plasmático é regulado pelos hormônios da paratireoide e pela calcitonina. Esses hormônios, além de aumentar o cálcio e reduzir o fósforo plasmático, aumentam a excreção do fosfato urinário, enquanto a calcitonina tem efeito contrário, inibindo a reabsorção óssea de cálcio e de fósforo. Quando os animais são submetidos a dietas pobres em fósforo, esse mecanismo fisiológico é acionado e ocorre a reciclagem do fósforo do osso. Neste contexto, a maior reciclagem de fósforo foi observada nos animais alimentados com a dieta controle.

De acordo com Koch et al. (1984), a exigência de fósforo para aumentar o desempenho dos animais é inferior ao nível exigido para máximo desenvolvimento dos ossos. Assim, em situações em que a dieta é pobre em fósforo, as necessidades fisiológicas dos animais são atendidas pela mobilização de fósforo dos ossos, levando os animais a crescerem com baixa resistência óssea. Esse mecanismo parece ser eficiente, porém, geralmente são mobilizados dos óssos quantidades superiores às necessárias para atender às necessidades dos animais, o que leva a maior excreção de fósforo nas fezes (Moreira et al., 2004 e 2009a).

O superfosfato simples e o fosfato bicalcico promoveram o menor fluxo de fósforo do plasma para os ossos e o menor refluxo do plasma para os ossos. A menor mobilização de fósforo do osso para o plasma, assim como dos tecidos para o plasma, comprova que essas fontes apresentam melhor

Tabela 3 - Fluxos de fósforo obtidos com as dietas pelos modelos de Vitti et al. (2000) e Lopes et al. (2001)

\begin{tabular}{|c|c|c|c|c|c|c|}
\hline \multirow[b]{2}{*}{ Modelo } & \multicolumn{6}{|c|}{ Fluxo entre os compartimentos (g/dia) } \\
\hline & $\begin{array}{c}\text { Trato } \\
\text { gastrointestinal } \\
\text { para plasma }\end{array}$ & $\begin{array}{c}\text { Plasma } \\
\text { para trato } \\
\text { gastrointestinal }\end{array}$ & $\begin{array}{c}\text { Plasma para } \\
\text { o osso }\end{array}$ & $\begin{array}{l}\text { Osso para } \\
\text { plasma }\end{array}$ & $\begin{array}{l}\text { Plasma para } \\
\text { tecidos moles }\end{array}$ & $\begin{array}{c}\text { Tecidos moles } \\
\text { para plasma }\end{array}$ \\
\hline Vitti et al. (2000) & $4,44 a$ & $0,77 \mathrm{a}$ & $4,48 a$ & $7,84 a$ & $5,48 a$ & $2,10 a$ \\
\hline Lopes et al. (2001) & $4,45 a$ & $0,79 a$ & $4,56 a$ & $4,03 b$ & $1,069 \mathrm{~b}$ & $0,90 b$ \\
\hline
\end{tabular}

${ }^{1}$ Médias seguidas por letras na coluna diferem pelo teste Duncan. 
ajuste ao metabolismo de suínos e são utilizadas mais eficientemente pelos animais.

As médias do fluxo de fósforo do trato gastrintestinal para o sangue, da fração do fósforo endógeno excretado no trato gastrointestinal e do fósforo do plasma para o osso não apresentaram diferenças. Todavia, os fluxos do plasma para os ossos e do plasma para os tecidos foram maiores pelo modelo de Lopes et al. (2001), enquanto o fluxo dos tecidos para o plasma foi maior pelo modelo de Vitti et al. (2000). As diferenças entre os modelos podem ser explicadas pelas características próprias desses modelos (Tabela 3).

No modelo de Vitti, os fluxos foram calculados por medidas diretas das atividades específicas (contagens radioativas e análises químicas), baseadas exclusivamente na experimentação científica. O modelo de Lopes apresenta uma estrutura com menor complexidade, sendo mais empírico e sujeito a erro experimental menor.

\section{Conclusões}

Os modelos apresentaram similaridade e eficiência na estimação do fluxo do fósforo nos compartimentos do sistema digestivo de suínos. O fosfato bicálcico apresenta melhor ajuste nos modelos e serve como referência para nutrição de suínos em crescimento.

\section{Referências}

BREVES, G.; SCHRODER, B. Comparative aspects of gastrointestinal phosphorus metabolism. Nutrition Research Reviews, v.4, p.125-140, 1991.

DAVIDSON, H.R. Pig meat: The carcass in relation to the live pig. In: DAVIDSON, H.R. (Ed.) The production and marketing of pigs. 3.ed. London: Longman, 1966. p.89-102.

FERNANDEZ, J.A. Calcium and phosphorus metabolism in growing pigs III, A model resolution. Livestock Prodution Science, v.41, n.1, p.2255-2261, 1995.

FIGUEIRÊDO, A.V.; VITTI, D.M.S.S.; LOPES, J.B. Disponibilidade biológica do fósforo de fontes fosfatadas determinada por intermédio da técnica de diluição isotópica para suínos em crescimento. Revista Brasileira de Zootecnia, v.30, n.5, p.1514-1520, 2001.

FISKE, C.H.; SUBBARROW, Y. The colorimetric determination of phosphorus. Journal Biological Chemistry, v.66, n.2, p.375-400, 1925.

INTERNATIONAL ATOMIC ENERGY AGENCY - IAEA. Laboratory training manual on the use of nuclear techniques in animal research. Vienna: IAEA, 1979. 299p. (Technical Report Series, 193).

JONGBLOED, A.W.; KEMME, P.A. Apparent digestible phosphorus in the feeding of pigs in relation to availability, requirement and environment. Digestible phosphorus in feedstuffs from plant and animal origin. Netherland Journal Agriculture Science, v.38, p.56-75, 1990.

KOCH, M.E.; MAHAN, D.C.; CORLEY, J.R. An evaluation of various biological characteristic in assessing low phosphorus in take in wealing swine. Journal of Animal Science, v.59, n.6 p.1546-1556, 1984.

LOPES, J.B. Efeito dos níveis de proteínas e energia sobre características de carcaça de suínos em crescimento e terminação. In: SEMINÁRIOS DE PESQUISA AGROPECUÁRIA DO PIAUÍ, 5., Teresina, 1988. Anais... Teresina: EMBRAPA, 1988. p.209-216.

LOPES J.B.; VITTI, D.M.S.S.; ABDALLA, A.L. et al. Modelo do fluxo biológico do fósforo de fontes de fosfato em suínos, usando o ${ }^{32} \mathrm{P}$ como marcador. Revista Brasileira de Zootecnia, v.30, n.1, p.165-173, 2001.

MOREIRA, J.A.; VITTI, D.M.S.S.; LOPES, J.B. et al. Fluxo biológico do fósforo no metabolismo de suínos alimentados com dietas contendo fitase. Revista Brasileira de Zootecnia, v.33, n.6, p.2066-2075, 2004.

MOREIRA, J.A.; VITTI, D.M.S.S.; TEIXEIRA, A.O. et al. Fisiologia digestiva de suínos alimentados com rações contendo diferentes fontes de fósforo. Revista Brasileira de Zootecnia, v.38, n.4, p.676-684, 2009a.

MOREIRA, J.A.; LOPES, J.B.; VITTI, D.M.S.S. et al. Modelos matemáticos para o estudo do fluxo biológico do fósforo em suínos alimentados com dietas suplementadas com níveis crescentes de fitase. Arquivo Brasileiro de Medicina Veterinária e Zootecnia, v.61, n.2, p.420-428, 2009b.

PERDOMO, C.C.; LIMA, G.J.M.M. Considerações sobre a questão dos dejetos e o meio ambiente. In: SOBESTIANSKY, S.; WENTZ, I.; SILVEIRA, P.R.S. et al. Suinocultura intensiva. Concórdia: EMBRAPA-CNPSA, 1998. cap. 11, p.221-235.

ROSTAGNO, H.S.; ALBINO, L.F.T.; DONZELE, J.L. et al. Tabelas brasileiras para aves e suínos. Viçosa, MG: UFV, 2005. $186 p$.

SARRUGE, J.R.; HAAG, H.P. Análises químicas em plantas. Piracicaba: ESALQ/USP, 1974. 56p.

SHENG, F.L.; WILLEM, C.S.; ARIE, K. et al. Effect of phytase supplementation to diets for weanling pigs on the utilization of phosphorus and calcium. Interciencia, v.31, n.4, p.262-267, 2006.

STATISTICAL ANALYSIS SYSTEM - SAS. The SAS system for windows. Release 8.01. Cary: SAS Institute, 2000. (CD-ROM).

TEIXEIRA, A.O.; LOPES, D.C.; RIBEIRO, M.C.T. et al. Composição química de diferentes fontes de fósforo e deposição de metais pesados em tecidos de suínos. Arquivo Brasileiro de Medicina Veterinária e Zootecnia, v.57, n.4, p.502-509, 2005.

TEIXEIRA, A.O.; LOPES, D.C.; LOPES, J.B. et al. Determinação da biodisponibilidade do fósforo de diferentes fontes pela técnica de diluição isotópica, em suínos em crescimento. Revista Brasileira de Zootecnia, v.33, n.5, p.1231-1237, 2004a.

TEIXEIRA, A.O.; LOPES, D.C.; VITTI, M.S.S. et al. Estimativas do fluxo de fósforo entre os compartimentos anatômicos e fisiológicos de suínos alimentados com dietas contendo diferentes fontes de fósforo. Revista Brasileira de Zootecnia, v.33, n.5, p.1246-1253, 2004b.

VITTI, D.M.S.S.; KEBREAB, E.; LOPES, J.B. A kinetic model of phosphorus metabolism in growing goats. Journal Animal Science, v.78, n.6, p.2706-2712, 2000.

VITTI, D.M.S.S.; ROQUE, A.P.; DIAS, R.S. et al. Metabolismo de cálcio em ovinos em crescimento sob suplementação com diferentes fontes de cálcio: aplicação e comparação de dois modelos matemáticos. Revista Brasileira de Zootecnia, v.35, n.6, p.2487-2495, 2006. 